\title{
Metodologías para la Evaluación de Activos Intangibles (pt. I)
}

\author{
Dr. Miguel A. Palomo González (*)
}

Resúmen: El valor de mercado de una empresa está determinado por los activos físicos y sus activos intangibles. Su expresión en el balance general permite que las empresas inicien un proceso de identificación de los activos intangibles y su cuantificación, para continuar con la administración del valor generado y su incremento en períodos futuros. La evaluación de los activos intangibles es una práctica administrativa del área de Administración de Tecnología que integra los conceptos de ingeniería financiera y de tecnología. En este artículo se presentan las diferentes metodologías de evaluación de los Activos Intangibles, con el fín de identificar el Modelo y su alcance ántes de su implantación.

Abstract: The two main components of the enterprise market value are the physical and intangible assets. Their presentation and amount in the enterprise's balance sheet is the starting point in a management process for intangible assets which continue with the value generation and improvement in future periods. The intangible assets valuation is a management practice in the Management of Technology function that compose financial engineering and technology concepts. This paper review different models to evaluate Intangible Assets, with the intent to identify the model and his scope before the implementation.

Palabras Clave (Keywords): Activos de Conocimiento, Activos Intangibles, Activos Intelectuales, Administración del Conocimiento, Capital Humano, Capital Intelectual, Tecnología.

\section{Introducción.}

A inicio de los noventas el concepto de "activos intangibles" [Al], como el de "la economía de servicios" y "la empresa del conocimiento", se consolidan en las Organizaciones. Es decir, los activos intangibles siempre han estado presentes en las organizaciones, el tema de su evaluación y su contribución al beneficio de la empresa es relativamente nuevo en la Administración de las empresas y disciplinas relacionadas. En México, en enero del 2002, el IMCP hace circular el Boletín de Activos Intangibles, donde se restringe el concepto a aquellos activos no circulantes que sin ser materiales o corpóreos son aprovechables en el negocio. Su incorporación al balance general no debe ser de manera subjetiva y depende de su capacidad de generar un beneficio.

Para dar una idea del concepto, si tratamos de evaluar una empresa lo mas evidente son los Activos Tangibles de la Empresa (Terrenos, Edificio, maquinaria y equipo, mobiliario, etc.) es decir los "fierros", estos determinan su Valor en Libros. Pero faltaría evaluar la parte "soft", los conocimientos humanos, el saber-hacer, las competencias del personal, la propiedad intelectual, las marcas, las relaciones con los clientes y los conocimientos sobre el comportamiento del mercado, los cuales son algunos ejemplos de Activos Intangibles y que (sumados al Valor en Libros) determinan el Valor de Mercado. Este artículo consta de dos partes, en la primera hablaremos de las metodologías, trataremos de dar respuesta a la pregunta: Qué se requiere para administrar y evaluar el Activo Intangible?, en la segunda parte hablaremos sobre los indicadores y las variables de medición del enfoque de evaluación.

\section{Cuál es la importancia de los activos intangibles?}

La Empresa Asset Equity Company (2002)menciona que, en 1985 el Valor en Libros representaba en promedio un 50 porciento del Valor de Mercado de las Empresas y que 15 años después, ahora, el Valor en Libros representa menos del $20 \%$ del Valor de Mercado de una Empresa, el 80 porciento restante es atribuido a los Al de la Empresa. Por su parte D. Skyrme (1997) menciona que en Junio de 1997, la

(*) El autor agradece a la Dirección y Sub-Dirección de Posgrado de FACPYA por el apoyo recibido para la presentación de esta ponencia en el Congreso de ACACIA, en la Mesa de Innovación y Tecnología (Acapulco, Gro., México, Mayo 5-7, 2004). El Dr. Miguel A. Palomo González es Profesor de Posgrado y del Programa de Doctorado en FACPYA, y de la 
Jefatura de Ingeniería Industrial, en la Facultad de Ciencias Químicas de la Universidad Autónoma de Nuevo León. e-mail: mpalomo@ccr.dsi.uanl.mx

relación del Valor de Mercado al Valor en Libros, para todas las empresas en el Industrial Dow Jones fue de 5.3, mientras que para varias de las empresas intensivas-en-conocimiento (ej: Microsoft, empresas Farmacéuticas) la relación fue de 10.0 .

En Mayo del 2002 la revista Expansión (2002) publica el artículo "Las Marcas más valiosas en México", apoyados por la metodología de evaluación de Marcas de la empresa inglésa Interbrand, y mencionan que el Valor en Libros de una empresa se estima en un tercio de su Valor de Mercado, "el resto reside en intangibles difíciles de medir, pero muy importantes en la toma de decisiones de inversionistas y administradores". En otra entrevista, David Clifton, Director de Interbrand México, menciona que el valor de la Marca puede representar el 80-90 porciento del Valor de una Empresa como en el caso de Chanel o Lacoste .(El Asesor de Monterrey, 2003)

\section{Cómo se define el activo intangible?}

En la literatura se utilizan indistintamente los términos de "Activos Intangibles", "Capital Intelectual", "Capital de Conocimiento" o "Activos Intelectuales", en la medida de lo posible nosotros utilizaremos el término de Activos Intangibles [Al] a lo largo del texto. Las principales observaciones son que la medición de retorno sobre activos $[R O A]$ y retorno sobre la equidad [ROE] no son realistas, pues no toman en cuenta los Al en los cálculos o no se reconocen en los beneficios de la empresa.

De acuerdo a B. Lev (Feng \& Lev, 2001) la empresa puede clasificar los factores que afectan su resultado económico en tres categorías:

- Activos físicos

- Activos financieros, $y$

- Activos Intangibles

Cada factor contribuye al beneficio en una cierta proporción, que sumados darían el total del beneficio de la empresa. La metodología identifica la proporción del beneficio que corresponde a los Al, generando un índice llamado "Beneficios del Capital de Conocimiento" (Knowledge capital earnings (C). Aunque no define "el contenido" completo de los Al, si logra determinar su impacto y es posible determinar el valor del capital que genera dicha proporción. Adicionalmente, resalta la importancia de administrar los Al; de los principales factores que impactan el capital intelectual como son la Investigación y Desarrollo, la Publicidad, la Información Tecnológica, y las prácticas de Recursos Humanos; finalmente, las mediciones identifican inversiones sobrevaluadas y sub-valuadas.

La Administración de los Al es un concepto relevante para la Administración de Tecnología y para la empresa, ya que no solo se refiere a administrar la fuerza de trabajo, ni trata de enfocarse al control del costo del producto o servicio, sino que busca el incremento de su valor y de los beneficios. En este sentido, la definición de Activos Intangibles se amplía a: el "conocimiento empacado", el cual incluye los procedimientos de la organización, tecnologías, patentes, habilidades de los empleados, y la información sobre los clientes, proveedores, e inversionistas. (Stewart T. A., 1997)(Luthy, 1998)

Edvinsson y Malone (1997) con un enfoque hacia la Administración de Activos Intangibles, presentan una clasificación de Capital Intelectual de la siguiente forma: 
- Capital Humano. Conocimientos, habilidades, y competencias de los empleados.

- Capital Estructural. La infraestructura que apoya la actividad humana (edificios, hardware, software, procesos, patentes, marcas, imagen de la organización, estructura organizacional, sistemas de información y bases de datos). Incluye los siguientes conceptos:

- Capital de Procesos. Técnicas, procedimientos, y programas que permiten la entrega de bienes y servicios.

- Capital de Innovación. Propiedad Intelectual (derechos de autor y marcas) y Activos Intangibles (definidos como: todos los talentos y teorías que hacen que una empresa funcione).

- Capital sobre Clientes. Es la fuerza y lealtad de las relaciones con los clientes (satisfacción de clientes, continuidad en los negocios, financieras, buenas relaciones, sensibilidad al precio).

La tabla siguiente nos da una clasificación más extensa sobre los Al (Brooking A., 1996):

\begin{tabular}{|c|c|c|c|}
\hline $\begin{array}{l}\text { Activos de } \\
\text { Mercado }\end{array}$ & $\begin{array}{l}\text { Activos de } \\
\text { Propiedad } \\
\text { Intelectual }\end{array}$ & $\begin{array}{l}\text { Activos } \\
\text { Humanos }\end{array}$ & \begin{tabular}{|l|} 
Activos de \\
Infraestruc- \\
tura
\end{tabular} \\
\hline $\begin{array}{l}\text { Marcas de } \\
\text { Servicios }\end{array}$ & Patentes & Educación & $\begin{array}{l}\text { Filosofía } \\
\text { administrativa }\end{array}$ \\
\hline $\begin{array}{l}\text { Marcas de } \\
\text { Productos }\end{array}$ & $\begin{array}{l}\text { Derechos de } \\
\text { autor }\end{array}$ & Calificaciones & $\begin{array}{l}\text { Cultura } \\
\text { Corporativa }\end{array}$ \\
\hline $\begin{array}{l}\text { Marcas } \\
\text { Corporativas }\end{array}$ & Diseños & sobre & $\begin{array}{l}\text { Procesos Ad- } \\
\text { ministrativos }\end{array}$ \\
\hline Clientes & Comerciales & & \\
\hline $\begin{array}{l}\text { Lealtad del } \\
\text { Consumidor }\end{array}$ & Saber-Hacer & $\begin{array}{l}\text { Evaluaciones de } \\
\text { puestos y } \\
\text { psicométricas }\end{array}$ & $\begin{array}{l}\text { Sistemas de } \\
\text { Información } \\
\text { Tecnológica }\end{array}$ \\
\hline $\begin{array}{l}\text { Continuidad de } \\
\text { Negocios. }\end{array}$ & Marcas & Competencias & $\begin{array}{l}\text { Sistemas de } \\
\text { Redes }\end{array}$ \\
\hline $\begin{array}{l}\text { Nombre de la } \\
\text { Empresa. }\end{array}$ & $\begin{array}{l}\text { Marcas de } \\
\text { Servicios }\end{array}$ & & $\begin{array}{l}\text { Relaciones } \\
\text { Financieras }\end{array}$ \\
\hline Backlog & & & \\
\hline Canales de & & & \\
\hline Distribución & & & \\
\hline Acuerdos de & & & \\
\hline Negocios & & & \\
\hline $\begin{array}{l}\text { Contratos de } \\
\text { Franquicias y } \\
\text { de Licencias }\end{array}$ & & & \\
\hline
\end{tabular}

Con el mismo enfoque de medir la contribución de los Al al beneficio de la empresa, A. Pulic (2000) propone un índice llamado el VAIC @. Su enfoque se centra sobre el valor agregado (EVAC) de las actividades y procesos, evalúa la contribución del capital empleado, del capital humano y del capital estructural (de acuerdo a los conceptos de Edvinsson y Malone) y junto con otros índices genera siete medidas de valor agregado, incluyendo 
el índice global VAIC@. El objetivo es identificar la creación de valor y la eficiencia de los Al en esa creación de valor. Las medidas son agregadas a nivel de la empresa y requiere del software VAICO.

Los enfoques para medir los $\mathrm{Al}$ dependen del interés particular de la empresa o de la información resultante a comunicar:

- evidenciar el valor de los Al en el valor actual del negocio,

- soportar el objetivo corporativo, de estar aumentando continuamente el valor de los accionistas,

- administrar los Al con valor y hacerlos crecer en el mediano y largo plazo,

- generar información mas útil para los potenciales inversionistas

y en función del enfoque será la metodología o modelo a emplear. En los enfoques anteriores se pretende cubrir el objetivo de conocer el valor generado por los Al, el valor en sí de los Al, administrar los Al en la creación de valor y aumentarlos, o simplemente para auditar y controlar los Al. Adicionalmente faltaría la clasificación de los procesos y actividades de los Al y los indicadores de medición. Por las características de los Activos Tecnológicos, la responsabilidad de su administración e implantación del modelo de medición recae en forma natural en el área de Tecnología de la empresa. La selección del modelo deberá ser en conjunto con la Dirección General y el primer nivel organizacional.

Entre los modelos o metodologías mas reconocidos para medir los Al se encuentran los siguientes:

Navegador Skandia (Skandia Navigator ( )): de la empresa de seguros Skandia, a la cual están relacionados Edvinsson y Malone, y que identifica los tres tipos mencionados de Capital Intelectual (Capital Humano, Capital Estructural y Capital del Consumidor), a su vez clasificados en 5 grupos, con 90 mediciones:

- Financieros (20)

- Humanos (13)

- Clientes (22)

- Procesos (16)

- Renovación/Desarrollo (19)

Valor Agregado Económico (Economic Value Added EVAC): esta metodología, desarrollada por Stern Stewart, se orienta a medir el retorno del capital empleado (ROA) del negocio. (Stewart T. A., 1997)

Balanced Scorecard: desarrollado por Kaplan y Norton (1996), esta diseñado para orientar a la Dirección o Ejecutivos del negocio hacia la identificación de los factores que contribuyen al éxito de la estrategia del negocio.

Monitor de Activos Intangibles (Intangible Assets Monitor): desarrollado por Karl Erik Sveiby divide los Activos Intangibles en Estructura Externa, Estructura Interna y Competencias de la Gente. Mide la creación de valor por los Al, en cuatro aspectos: crecimiento, renovación, utilización/eficiencia, y reducción del riesgo/estabilidad.

Indice del Capital Intelectual (Intellectual Capital Index IC-Index ( )): desarrollado por la empresa Intellectual Capital Services. Es un índice global que indica los cambios en el valor de mercado de la empresa.

Metodología del Valor Incluyente (Inclusive Value Methodology): desarrollada por el profesor Philip McPherson. Calcula un Valor Agregado combinado, formado por el valor agregado monetario y el valor agregado de los activos intangibles.

\section{Cómo se clasifican los enfoques o modelos para medir los activos intangibles?}


Dependiendo del objetivo o información a comunicar y el nivel de análisis requerido (a nivel de negocio, de sus componentes, procesos y/o actividades), podemos clasificar los métodos actuales de evaluación de los Al, en las siguientes categorías (Sveiby, 2002):

- Métodos del Capital Intelectual Directo (Direct Intellectual Capital Methods) [DIC]: estiman el valor financiero del activo intangible global a partir de cada uno de sus componentes.

- Métodos de Capitalización de Mercado (Market Capitalization Methods) [MCM]: calculan la diferencia entre la capitalización de mercado de una empresa y el valor de sus activos (tangibles), siendo esta diferencia el valor del capital intelectual o activos intangibles.

- Métodos del Retorno sobre Activos (Return on Assets Methods) [ROA]: el promedio de los beneficios antes de impuestos, en un período de tiempo, es dividido por el promedio de los activos (tangibles) de una empresa. El resultado es el ROA de la empresa, el cual es comparado con el promedio de la industria, la diferencia con el ROA de la industria nos da el ROA (\%) generado por los activos intangibles; este a su vez se multiplica por los activos tangibles promedio, para calcular el beneficio promedio generado por los intangibles. Posteriormente al dividir los beneficios promedio de los activos intangibles, por una tasa de interés o costo de capital para la empresa, se obtiene el valor de los activos intangibles (principal) que generó el beneficio promedio (diferencial).

- Scorecard Methods [SC]: Una vez identificados los componentes del activo intangible, o capital intelectual, entonces se genera indicadores o índices que se reportan en un tablero para su seguimiento. Los métodos SC son similares a los métodos DIC, la diferencia es que no se le da un valor monetario al activo intangible. Un índice global puede o no ser calculado.

La ventajas de los métodos MCM y ROA es que ofrecen una evaluación monetaria, y por lo tanto son recomendables para el caso en que exista interés en el negocio por una fusión y/o adquisición, o para valuar las acciones (stock) en el mercado.

La ventaja de los métodos DIC y SC es que generan una fotografía completa de los Al, se pueden aplicar a cualquier nivel de la organización, y se pueden adaptar fácilmente a las organizaciones públicas o sociales puesto que no se requiere de una medición financiera.

Debemos tener claro el objetivo de medir los Al, entre más claro sea el modelo más fácil será su implantación a nivel de la organización, a nivel de procesos y/o actividades a medir, y la identificación de los indicadores a medir. Recordemos que algunos indicadores serán no-financieros y adaptados a una organización específica, por lo tanto serán difíciles de comparar, otros serán financieros y fáciles de aceptar y comunicar. La fig.1 nos muestra una clasificación de los Métodos de Medición de Al dependiendo de su aplicación y medición.

\section{(Incertar Fig. 1: Modelos de Medición de los Activos Intangibles) ( Fig-1 Modelos AI.TIFF)}

Entonces, para cada negocio, nivel de la organización, o situación de mercado, dependerá el enfoque, metodología o modelo para medir los Al. Los Principales enfoques y su razón de ser se pueden clasificar de acuerdo a la siguiente tabla y extender su aplicación a otros niveles de la organización.

\begin{tabular}{|l|l|}
\hline \multicolumn{1}{|c|}{ Enfoque: } & \multicolumn{1}{c|}{ Razón de ser: } \\
\hline Reporte de Accionistas & Justificación \\
\hline Monitoreo de Resultados & Control \\
\hline Adquisiciones /Nenta & Evaluación del Negocio \\
\hline Guía para Invertir & Decisión \\
\hline
\end{tabular}


En esta primera parte podemos concluir que la selección del Modelo de Evaluación de Activos Intangibles depende de:

- la determinación del Objetivo. Por qué medir?

- definir si evaluamos el valor agregado de los Al, el valor del Al, o ambos. Qué queremos evaluar?

- la definición de los niveles de medición, índice global, índice de los componentes medulares del negocio. Cuál debe ser el alcance?(Direcciones, Gerencias y Jefaturas).

Pero, sin un buen Sistema de Información Interno y Externo, corremos el riesgo de generar una enorme cantidad de "sabanas" con datos abundantes, difíciles de analizar, de integrar, y difíciles de explicar su significado 0 variabilidad; de igual forma si el área de Tecnología no es la responsable de la administración del modelo de medición, corremos el riesgo de medir lo irrelevante para el negocio y que se convierta en un modelo estéril, sin continuidad en el tiempo y vulnerable a los cambios organizacionales. En la segunda parte de este artículo comentaremos sobre los indicadores, mediciones y factores que determinan la operación exitosa del Modelo de Evaluación de los Activos Intangibles.

\section{Referencias:}

El Asesor de Monterrey, 2003. El valor de las marcas. Abril 21- Mayo 4: 9.

Assets Equity Company, 2002. Measuring Intangible Equity. 2p. Info@assetequity.com

Brooking A., 1996. Intellectual capital: core asset for the third millennium enterprise. International Thomson Business Press, New York (in: Luthy, 1998)

Edvinsson L. \& Malone M. S., 1997. Intellectual capital: realizing your's true value by finding its hidden roots. Harper Collins Publishers Inc., N.Y. (in: Luthy 1998).

Expansión, 2002. Las marcas más valiosas en México. 15-29 Mayo: 38-54.

Gu F. \& Lev B., 2001. Intangible assets: measurement, drivers, usefulness. Boston University \& New York University, $47 \mathrm{p}$. http://pages.stern.nyu.edu/ blev/

Kaplan R. S. \& Norton D. P., 1996. Using the balanced scorecard as a strategic management system. HBR, Jan-Feb: 75-85. www.balancedscorecard.com

Luthy D. H., 1998. Intellectual capital and its measurement. www3.bus.Osaka-cu.ac.jp/apira98/ archives/ htmls/25.htm

Pulic A., 2000. An accounting tool for IC management. www.measuring-ip.at/papers/ham99txt.htm

Skyrme David 1997. Measuring Intellectual Capital. Insight No. 24, 5p. www.skyrme.com

Stewart T. A., 1997. Intellectual capital. Doubleday, N.Y. 1997, (in: luthy, 1998). www.sternstewart.com

Sveiby K-E., 2002. Methods for measuring intangible assets. Oct.: 5p. www.sveiby.com 


\section{Fig. 1: Modelos de Medición de los Activos Intangibles}

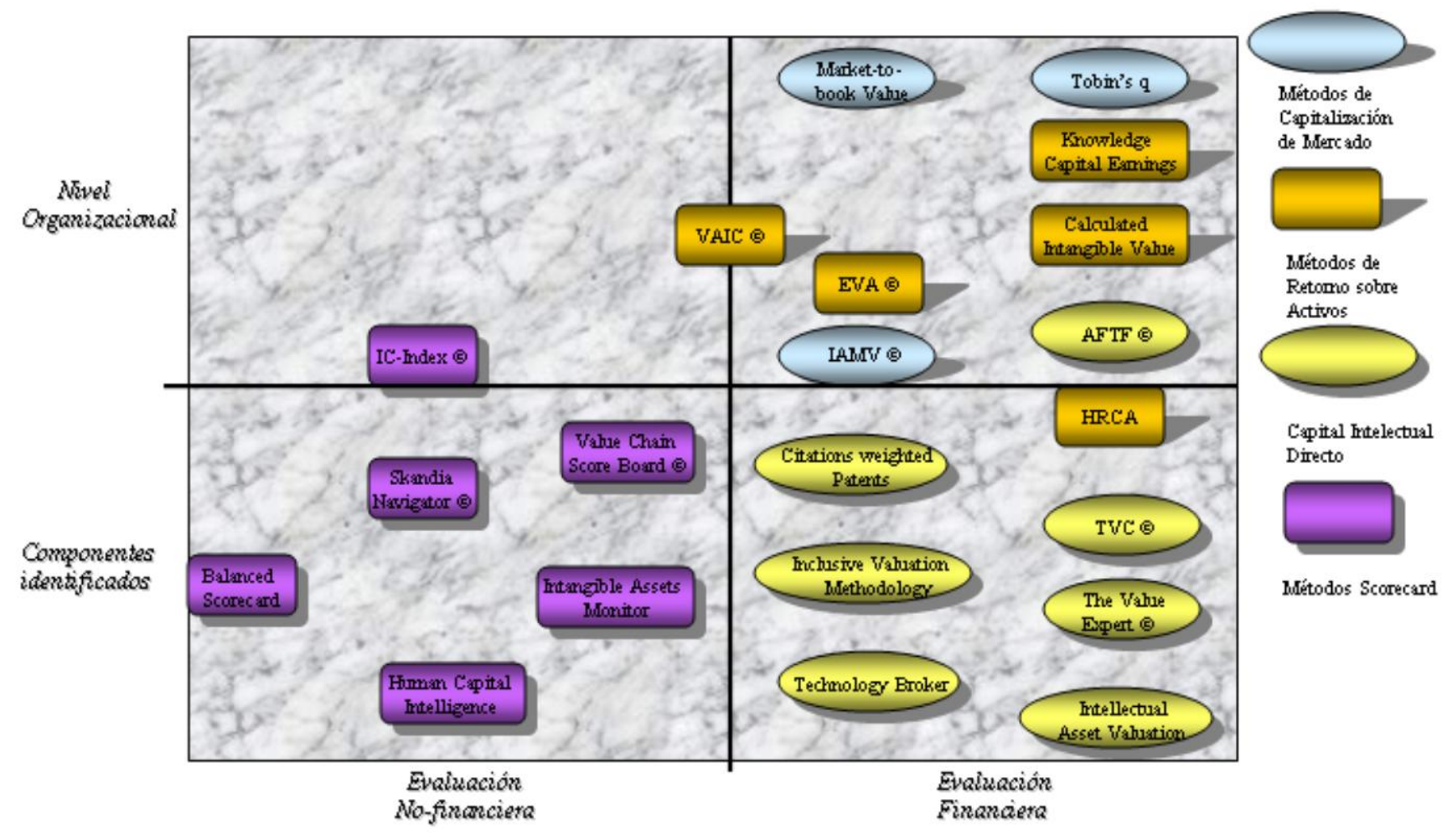

So: Adaptado de Karl-Erik Sve by, Oct. 2002. 